\title{
Editorial: Zukunft der Arbeitszeit
}

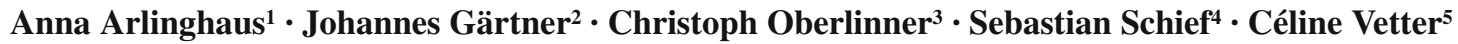

Online publiziert: 17. März 2016

(C) Springer-Verlag Berlin Heidelberg 2016

Liebe Leserinnen, liebe Leser,

Prognosen sind schwierig, besonders wenn sie die Zukunft betreffen. Dieses Bonmot wird wahlweise dem Physiker Niels Bohr, der Ikone bayerischen Humors Karl Valentin oder dem Schriftsteller Mark Twain zugeschrieben. Unabhängig von der Herkunft dieses Zitates spricht es ohne Zweifel einen wichtigen Punkt jeglicher Forschung an: Inwiefern können wir aus den Analysen der Vergangenheit und Gegenwart Schlüsse für die Zukunft ziehen? Die Arbeitszeitgesellschaft hat sich in ihrem 2. Symposium am 26. September 2014 in den Räumen der Bundesanstalt für Arbeitsschutz und Arbeitsmedizin (BAuA) in Dortmund der Frage der Zukunft der Arbeitszeit gewidmet und versucht, auf der Basis der Arbeitszeitforschung Folgerungen für die Zukunft zu ziehen. Die Arbeitszeitgesellschaft e. V. wurde im Jahr 2013 gegründet und hat sich als gemeinnütziger Verein zum Ziel gesetzt, die interdisziplinäre Erforschung der Arbeitszeitgestaltung, inklusive ihrer Auswirkungen, Gefahren und Möglichkeiten zur Verbesserung, zu

A. Arlinghaus

arlinghaus@ximes.com

1 XIMES GmbH, Wien, und GAWO e.V., Mainz, Deutschland

2 XIMES GmbH und TU-Wien, Wien, Österreich

3 BASF SE, Ludwigshafen, Deutschland

4 Departement für Sozialwissenschaften, Universität Fribourg, Fribourg, Schweiz

5 Channing Division of Network Medicine, Brigham's and Women's Hospital, Harvard Medical School, Boston, USA fördern. Auch soll ein Forum für die Zusammenarbeit zwischen Wissenschaft und Praxis geschaffen werden.

Die Teilnehmer des Symposiums stammten sowohl aus verschiedenen wissenschaftlichen Disziplinen als auch aus unterschiedlichen betrieblichen Bereichen und aus Interessenvertretungen. Dieses Themenheft beinhaltet eine Auswahl der auf dem Symposium vorgestellten Beiträge.

Die 12 Artikel drehen sich im Wesentlichen um drei Themenbereiche: Ein erster Block verhandelt verschiedene Aspekte der Schichtarbeit. Der zweite Block diskutiert neue Arbeitszeitmodelle und ihre Auswirkungen auf die Beschäftigten. Im letzten Block werden Fragen der Messung und Verteilung von Arbeitszeit und Arbeitszeitwünschen diskutiert, insbesondere in Bezug auf Unterschiede zwischen Männern und Frauen.

Im ersten Teil des Sonderheftes werden praktische Beispiele der Schichtarbeit und ihre Auswirkungen analysiert. Der Beitrag von Scherf und Karras zeigt, wie mit flexiblen Schichtplänen Lösungen generiert werden können, die sowohl die betriebliche Bedarfsdeckung verbessern als auch die Verlässlichkeit der Freizeit für die Mitarbeiter deutlich erhöhen. Die Autoren zeigen auf, wie ein verbessertes Schichtsystem in Zukunft aussehen könnte.

Gärtner, Marschitz, Baumgartner und Boonstra-Hörwein zeigen anhand verschiedener Praxisbeispiele, dass entgegen einem stereotypischen Bild von Schichtarbeit häufig substanzielle Spielräume der Zeiteinteilung existieren, die für neue Arbeitszeitmodelle innerhalb der Schichtarbeit genutzt werden können. Das gilt insbesondere für die Gleitzeit, die derzeit noch primär im Bürobereich genutzt wird.

Oberlinner, Halbgewachs und Yong zeigen anhand einer eigens durchgeführten Befragung, wie sich verschiedene Arbeitsformen auf den so genannten Work-Ability-Index auswirken. Die Ergebnisse verweisen auf geringe negative Auswirkungen auf den Gesundheitszustand der Mitarbeite- 
rinnen und Mitarbeiter durch das im untersuchten Unternehmen angewandte Wechselschicht-System.

Rott und Watrinet berichten von einer Schichtplanumstellung in einem Unternehmen und deren Auswirkungen. Das Unternehmen hat ein unregelmäßig kurz rotierendes, vollkontinuierliches Schichtmodell auf Basis vollzeitnaher Teilzeit eingeführt, das - dank einer wöchentlichen SollArbeitszeit von 31,5 Stunden - eine vollzeitnahe Teilzeit in Schicht und die im Tarifvertrag Chemie vorgesehene Altersfreizeit erlaubt. Ergebnisse einer Mitarbeiterbefragung verweisen darauf, dass die Zufriedenheit und Motivation der Mitarbeiterinnen und Mitarbeiter gestiegen ist.

Windscheid und Chodora diskutieren die Akzeptanz ergonomisch gestalteter Schichtpläne anhand einer Evaluationsstudie in der bayerischen Metall- und Elektroindustrie. Ihre Befragung in fünf Werken zeigt auf, dass die Mehrheit der Befragten ergonomische Schichtpläne akzeptiert, allerdings ist eine gewisse Eingewöhnungszeit erforderlich. Insbesondere eine hohe Schlafqualität, größere Chancen auf soziale Teilhabe und vorweg eine adäquate Information und Beteiligung der Akteure erhöhte die Akzeptanz der Schichtpläne.

Der Frage, ob bestimmte neue Arbeitszeitmodelle Vorteile für Unternehmen und Beschäftigte zeitigen, geht Haidacher nach. Sie beschreibt anhand der Innsbrucker Verkehrsbetriebe, wie so genannte Mischarbeit zu guten Arbeitsbedingungen führen und längere Berufstätigkeit fördern kann. Zudem können durch diese Arbeitsform die Zufriedenheit der Mitarbeiterinnen und Mitarbeiter erhöht und saisonbedingte Auslastungsschwankungen besser gestaltet werden.

Scherz und Schwendinger diskutieren, inwiefern betriebliche Arbeitszeitpolitik, genauer eine neue kollektivvertragliche „Freizeitoption“, die Work-Life-Balance verbessern können. Anhand einer Befragung können sie zeigen, dass das Interesse an dieser Option breit gefächert ist und die Freizeitoption als langfristiges Arbeitszeitkonto Vorteile mit sich bringt.

Griesbacher widmet sich der Frage, welche soziostrukturellen Rahmenbedingungen die zeitlichen Selbstbestimmungsmöglichkeiten unselbständig Beschäftigter bei flexiblen Arbeitszeiten beeinflussen können. Er kann aufzeigen, welche Folgen zeitliche Selbstbestimmung haben kann und betont, dass betriebliche Kommunikation über die Möglichkeiten der Inanspruchnahme von zeitlicher Selbstbestimmung von enormer Bedeutung ist. Zeitliche Selbstbestimmung sei demnach, so Griesbacher, keine lediglich individuelle Angelegenheit.

Kümmerling und Lazarevic beleuchten, wie Messkonzepte und Berechnungsmethoden der Arbeitszeit zu Arte- fakten führen können. Der Autor und die Autorin zeigen in einem ersten Teil, welchen Einfluss die Art der Fragestellung auf die Resultate von Bevölkerungsumfragen zum Thema Arbeitszeit hat. Insbesondere zeigen sie, dass in diesem Bereich ein großer Unterschied zwischen Männern und Frauen besteht. Im zweiten Teil werden Vollzeitäquivalenzraten als arbeitszeitbasierte Maßzahl der Erwerbstätigkeit betrachtet und ihr Nutzen für Gruppenvergleiche der Arbeitsmarktinklusion diskutiert.

Wanger gibt einen empirischen Überblick über Erwerbsund Arbeitszeitmuster in Paarbeziehungen. Sie kann zeigen, dass die Erwerbsbeteiligung von Frauen in den letzten Jahren zwar kontinuierlich angestiegen ist, aber der hohe Anteil an Frauen, die Teilzeit arbeiten, zu einer ausgeprägten Arbeitszeitlücke zwischen Männern und Frauen führt. Es zeigt sich, dass insbesondere bei Paaren mit Kindern die Unterschiede im zeitlichen Ausmaß der Erwerbsarbeit stabil sind. Selbst die Realisierung der Arbeitszeitwünsche würde in Bezug auf die Verteilung der partnerschaftlichen Erwerbszeit wenig ändern.

Sopp und Wagner untersuchen die Arbeitszeitwünsche von Männern und Frauen und deren Realisierungswahrscheinlichkeit anhand des Sozio-ökonomischen Panels (SOEP). Sie interessiert, inwiefern sich seit der Einführung des Teilzeit- und Befristungsgesetzes im Jahr 2001 sowie anderer Gesetze Wirkungen in Richtung kürzerer Arbeitszeiten nachweisen lassen. Die durch die Gesetze intendierten Wirkungen ließen sich laut Sopp und Wagner nicht belegen. Es zeigte sich jedoch, dass Wünsche nach Beibehaltung der vertraglichen Arbeitszeiten im Zeitverlauf eine größere Realisierungschance erhielten. Dies könnte ein Hinweis auf die zunehmende Verbreitung bzw. Wirkung von interner Arbeitszeitflexibilisierung sein.

Schief resümiert in seinem Kurzbeitrag mehrere Forschungen zum Einfluss politischer und institutioneller Rahmenbedingungen auf die Zeitungleichheit von Männern und Frauen in bezahlter und unbezahlter Arbeit. Die Genderzeitungleichheit in der Schweiz wird von ihm analysiert, anschließend macht er politisch-institutionelle Vorschläge zur Verringerung dieser Ungleichheit. Insbesondere die langen Arbeitszeiten von Männern stellen seiner Einschätzung nach ein massives Problem für die Überwindung der Zeitungleichheit dar.

Das 2. Symposium der Arbeitszeitgesellschaft lieferte spannende Einsichten über verschiedene mögliche Entwicklungen im Bereich der Arbeitszeit. Wir hoffen, Anstöße sowohl für die weitere Forschung als auch für die Praxis der Arbeitszeitorganisation gegeben zu haben. Die Arbeitszeitgesellschaft hat inzwischen ein 3. Arbeitszeit- 
symposium in Wien abgehalten, das nächste wird am 7. Oktober 2016 in Dortmund stattfinden. Wir freuen uns auf Ihre Beiträge und Reaktionen und möchten Sie dazu schon jetzt herzlich einladen!
Eine spannende Lektüre wünscht Ihnen der Vorstand der Arbeitszeitgesellschaft e. V.

Anna Arlinghaus, Johannes Gärtner, Christoph Oberlinner, Sebastian Schief, Céline Vetter 